\title{
Clinical Advantage of Chest-wall Post-mastectomy Radiation Therapy Without Bolus
}

\author{
SHINTARO SHIBA ${ }^{1}$, MASAHIKO OKAMOTO $^{1}$, HIROKI KIYOHARA ${ }^{2}$, NAOKO OKANO $^{1}$, YUYA YOSHIMOTO $^{1}$, \\ HIROTO MURATA ${ }^{3}$, DAISUKE IRIE ${ }^{4}$, HIROYUKI KATOH ${ }^{5}$ and TAKASHI NAKANO ${ }^{1}$ \\ ${ }^{1}$ Department of Radiation Oncology, Gunma University Graduate School of Medicine, Gunma, Japan; \\ ${ }^{2}$ Department of Radiation Oncology, Japanese Red Cross Maebashi Hospital, Gunma, Japan; \\ ${ }^{3}$ Hospital, National Institute of Radiological Sciences, \\ National Institutes for Quantum and Radiological Science and Technology, Chiba, Japan; \\ ${ }^{4}$ Department of Radiation Oncology, Nara Medical University Graduate School of Medicine, Nara, Japan; \\ ${ }^{5}$ Department of Radiation Oncology, Kanagawa Cancer Center, Yokohama, Japan
}

\begin{abstract}
Background/Aim: The clinical outcomes of postmastectomy radiation therapy (PMRT) without bolus remain to be fully examined, so that we evaluated clinical outcomes of PMRT without bolus and to measure the chest-wall dose surface histogram (DSH) parameters. Patients and Methods: Fifty-two patients with breast cancer who received PMRT without bolus were retrospectively analyzed. DSH values of the percentage of maximum dose $\left(D_{\max }\right)$ were measured. Results: All patients completed the treatment; the median follow-up period was 22.1 months. The 2-year overall survival and local control rates were $85 \%$ and $95 \%$. Five patients developed grade 2 acute radiation dermatitis, and none developed grade 2 or higher late radiation dermatitis. The median $D_{\max }$ in patients who developed grade 0-1 and grade 2 acute radiation dermatitis was 5,178 and 5,365 cGy ( $p=0.03)$. Conclusion: PMRT without bolus resulted in a low frequency of grade 2 or higher radiation dermatitis without increasing locoregional recurrences, and the $D_{\max }$ was the contributing factor for developing acute radiation dermatitis.
\end{abstract}

Post-mastectomy radiation therapy (PMRT) improves local control and survival in patients with breast cancer with a high risk of recurrence (those with advanced tumor and/or multiple lymph node metastases). PMRT reduces locoregional and distant recurrence in patients with axillary

This article is freely accessible online.

Correspondence to: Masahiko Okamoto, Department of Radiation Oncology, Gunma University Graduate School of Medicine, 3-3922 Syowa-machi, Maebashi, Gunma 371-8511, Japan. Tel: +81 272208383, Fax: +81 272208397, e-mail: okamott@gunma-u.ac.jp

Key Words: Breast cancer, dermatitis, radiation therapy, mastectomy. dissection and in those with axillary sampling, and radiation dermatitis is its most problematic adverse event (1). Almost all reports have used a bolus to maximize the radiation dose for the chest-wall surface and to decrease the risk of local recurrence, disregarding the radiation dermatitis problem (2). However, the use of a bolus has been reported to increase the frequency of severe radiation dermatitis (3). Few reports have compared the clinical outcomes of PMRT between patients using a bolus and those not using one, and no reports have analyzed the chest-wall dose surface histogram (DSH) parameters for PMRT. Therefore, a retrospective analysis of clinical outcomes in PMRTs without bolus was conducted and the DSH parameters between irradiation plans with and without virtual boluses were compared.

\section{Patients and Methods}

Patients. Between October 2011 and December 2016, 55 patients with breast cancer received PMRT at the Gunma University Hospital. Among these patients, three who received boost irradiation with electron beams were excluded because of the difficulty in recreating the precise dose distribution of electron beams using our radiation treatment planning system (RTPS) of the XiO version 4.5 software (ELEKTA, Stockholm, Sweden). Thus, 52 patients with breast cancer who received PMRT were retrospectively analyzed, and all patients were treated with $6 \mathrm{MV} \mathrm{X}$-ray beams without bolus. Patient characteristics are summarized in Table I. Histologically, 46 patients had invasive ductal carcinoma, three had invasive lobular carcinoma, and four had other breast cancer types. Breast cancer subtypes were defined according to the status of estrogen receptor (ER), progesterone receptor (PgR), human epidermal growth factor receptor 2 (HER2), and the Ki-67 index. Twenty-four patients had luminal A (ER-positive and/or PgR-positive, HER2-negative, and Ki-67<20\%), 20 had luminal B (luminal type other than luminal A), four had HER2-enriched (ER-negative, PgR-negative, and HER2-positive), and four had triple-negative (ER-negative, PgRnegative, and HER2-negative) cancer subtypes. Twelve patients had 
clinical and/or pathological T4 components and 36 had four or more pathologically proven lymph node metastases. The present study complied with the standards of the Declaration of Helsinki and current ethical guidelines and all patients signed an informed consent form before the initiation of therapy.

Anticancer drug therapy. Twenty-four patients received anticancer drug therapy before surgery. Among these, 16 received 5-fluorouracil, epirubicin, and cyclophosphamide (FEC) followed by taxane regimen, five received FEC followed by taxane and trastuzumab regimen, and three received other regimens.

Twenty-three patients received anticancer drug therapy during the period between surgery and PMRT. Among these, 10 received FEC followed by taxane regimen, five received FEC followed by taxane and trastuzumab regimen, three received FEC followed by taxane and endocrine therapy regimen, and five received other regimens.

Fifty patients received anticancer drug therapy after PMRT. Among these, 27 received endocrine therapy alone, eight received endocrine therapy and tegaful-uracil, two received FEC followed by taxane regimen, three received endocrine therapy and trastuzumab, three received trastuzumab alone, and seven received other regimens.

Radiation therapy. All patients received total mastectomy and PMRT without bolus. Sets of 2.5-mm-thick CT images were acquired for treatment planning. The three-dimensional treatment planning was performed using the XiO RTPS.

Forty-nine patients received chest wall and supraclavicular and infraclavicular lymph node irradiations and three received only chest wall irradiation. The total dose to the chest wall and/or supraclavicular and infraclavicular lymph node regions was $50 \mathrm{~Gy}$ in 25 fractions with $6 \mathrm{MV} \mathrm{X}$-ray beams. Figure 1 shows a typical radiation field with dose distribution.

Dose surface histogram analyses. There are various methods for skin area definition in RTPS. a skin surface thickness of $1.0 \mathrm{~mm}$ was chosen (4), and the skin structures were automatically contoured using the $\mathrm{XiO}$ software. A 5-mm tissue-equivalent structure on each patient's surface was used as a virtual bolus in our simulated plan. The dose distributions for the skin structures without bolus (real plan) and with virtual bolus (simulated plan) were calculated on the Plan-CT. DSH parameters of the percentage of maximum dose $\left(D_{\max }\right)$, the doses to $2-\mathrm{cm}^{3}$ volumes $\left(\mathrm{D}_{2 \mathrm{cc}}\right)$, and the skin areas that received at least $5,10,20,30$, or $40 \mathrm{~Gy}\left(\mathrm{~S}_{5}, \mathrm{~S}_{10}, \mathrm{~S}_{20}, \mathrm{~S}_{30}\right.$, or $\left.\mathrm{S}_{40}\right)$ in the real and simulated plans were measured and analyzed.

Evaluation during follow-up and statistical analysis. After the completions of PMRT, patients were mainly followed up at our hospital. The follow-up examinations comprised interviews, physical examinations, routine blood cell counts, and blood chemistry and diagnostic imaging tests such as mammography, ultrasonography, CT images, and magnetic resonance images. Acute and late toxicities were classified using the National Cancer Institute's Common Terminology Criteria for Adverse Events, version 4.0 (5). Acute toxicity was defined as the highest toxicity within 3 months from the initiation of PMRT, and late toxicity was defined as the highest toxicity after 3 months from the initiation of PMRT. Survival duration was measured from the date of initiation of PMRT to the date of death or to the most recent followup, and disease-free survival (DFS) was measured from the initiation of PMRT to the date of the first tumor progression disease. Locoregional recurrence was defined as tumor regrowth in the
Table I. Patient characteristics $(n=52)$.

Characteristics

Age in years, median (range)

$58(34-75)$

Histology, number of cases

Invasive ductal carcinoma

$46(88.4 \%)$

Invasive lobular carcinoma

$3(5.8 \%)$

Others

$3(5.8 \%)$

Subtype, number of cases

Luminal A

$24(46.2 \%)$

Luminal B

$20(38.4 \%)$

HER2-enriched

$4(7.7 \%)$

Triple-negative

$4(7.7 \%)$

$\mathrm{T} 4$ component, number of cases

Yes

$12(23.1 \%)$

No $\quad 40(76.9 \%)$

Number of pathological lymph node metastases

$0-3$

$16(30.7 \%)$

4-10

$24(46.2 \%)$

$\geq 11$

$12(23.1 \%)$

HER: Human epidermal growth factor receptor.

irradiated field. The probabilities of overall survival (OS), locoregional control (LC), and DFS rates were calculated using the Kaplan-Meier method, and the Wilcoxon signed rank test and Student's $t$-test were used for statistical analysis of differences in DSH parameters. Receiver operating characteristic (ROC) curves to determine the optimal cutoff values for $D_{\max }$ were measured to identify severe dermatitis cases. The cutoff value was defined according to the nearest point from the coordinates $(0,1)$ on the ROC graph. All statistical analyses were performed using the SPSS Statistics version 23 software (IBM Institute, Tokyo, Japan). $p<0.05$ was considered statistically significant.

\section{Results}

Clinical outcomes. All patients completed the planned PMRT, and the median follow-up period since the initiation of irradiation was 22.1 (range=5.1-58.7 months) months. At the time of analysis, two (4\%) patients had locoregional recurrence; one of these patients had a supraclavicular lymph node recurrence and the other had skin recurrence at the surgical wound site. Six (12\%) patients died of breast cancer. The 2-year estimated OS, LC, and DFS rates after PMRT were $85 \%, 95 \%$ and $81 \%$, respectively (Figure 2 ). With regard to toxicities, five $(10 \%)$ patients developed grade 2 acute radiation dermatitis and none developed grade 2 or higher late radiation dermatitis and symptomatic radiation pneumonitis.

DSH parameter analyses for real and simulated RTPS plans. DSH parameters with and without virtual boluses were analyzed, and the results are shown in Table II. There were significant differences in all measured DSH parameters between the real and simulated plans, and $p$-values were all statistically significant. 


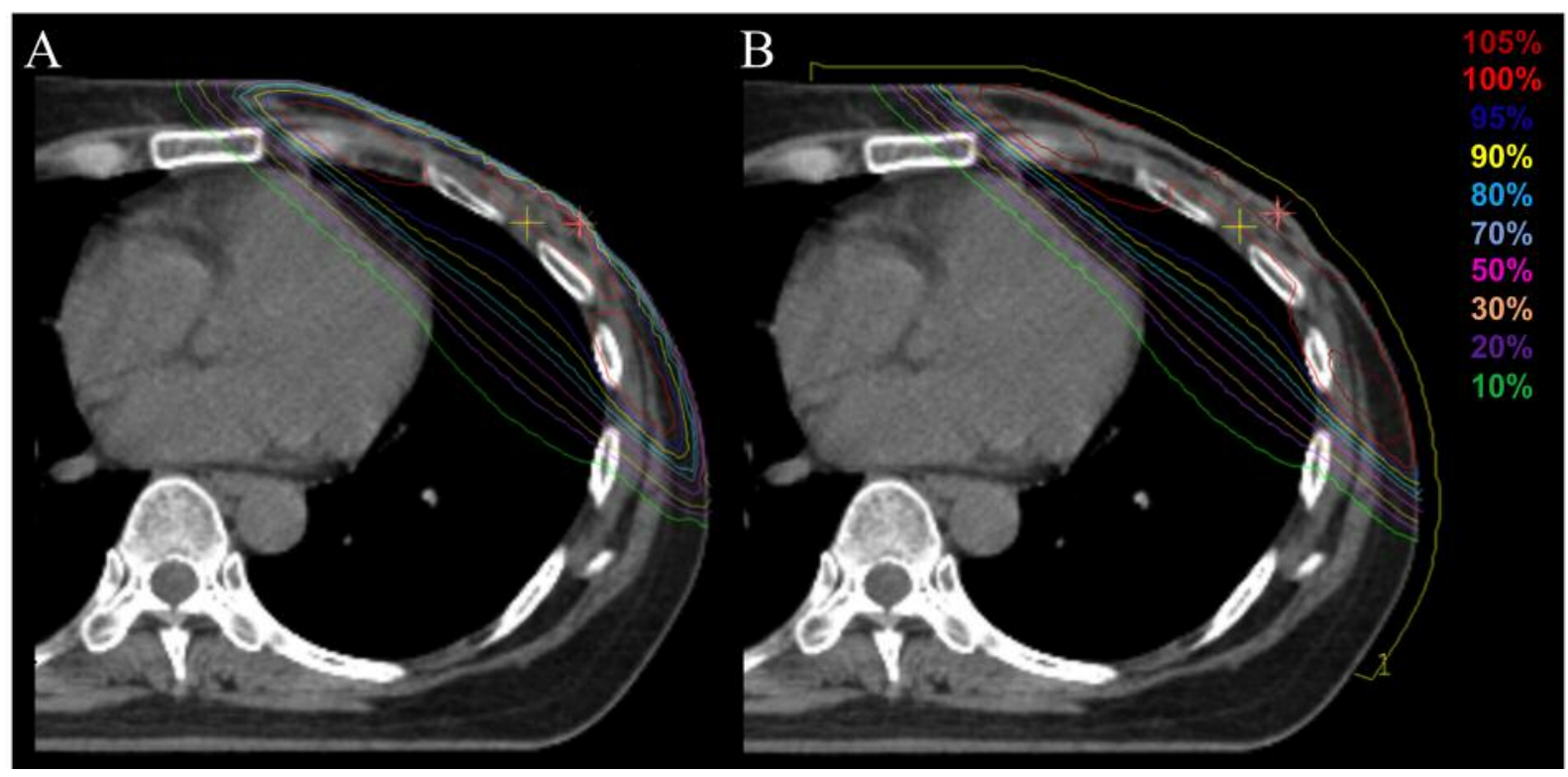

Figure 1. Representative patient axial CT images with and without virtual bolus. Dose distribution on axial CT images of PMRT without bolus is shown in (A) and that on axial CT images of PMRT with virtual bolus is shown in (B). Yellow line on the body surface represents the 5-mm skin bolus.

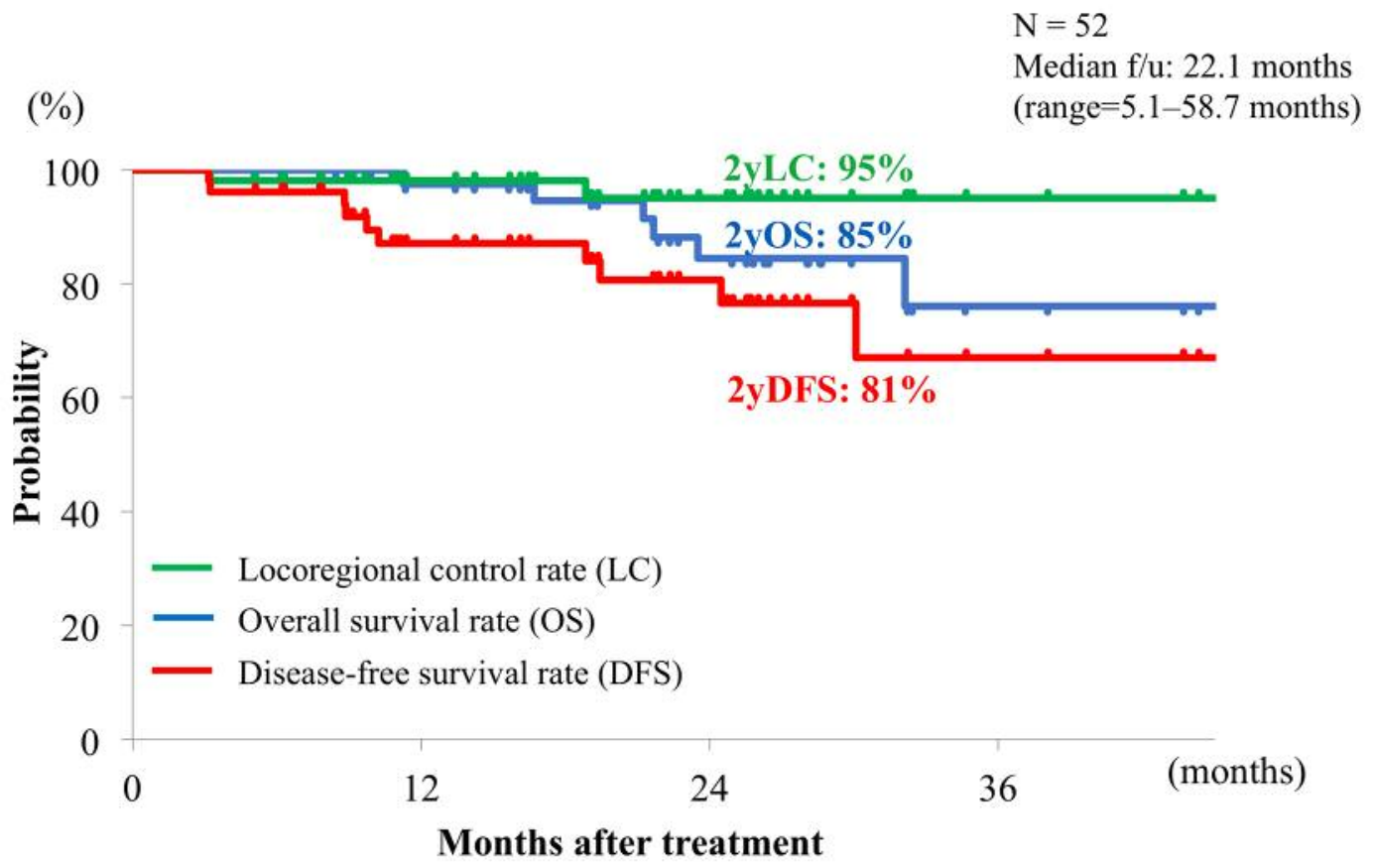

Figure 2. Graph showing survival rates for patients treated with PMRT. OS (blue line), local control (green line), and DFS (red line) are shown for all patients treated with PMRT.

Correlation between DSH parameters and dermatitis. For patients who received PMRT without bolus, the median skin $\mathrm{D}_{\max }$ was $5178 \mathrm{cGy}$ (range=4861-5618 cGy) in patients who developed grade $0-1$ acute radiation dermatitis and $5365 \mathrm{cGy}$ (range $=5,160-5,478 \mathrm{cGy}$ ) in patients who developed grade 2 acute radiation dermatitis, and the difference was statistically significant $(p=0.03)$.

When the ROC curves for $\mathrm{D}_{\max }$ of PMRT in the real plan were analyzed, the area under the curve was 0.817 and the optimal cutoff values for discriminating the development of 
Table II. Dose surface histogram parameters of planned PMRTs with and without virtual boluses $(n=52)$.

\begin{tabular}{lccc}
\hline Variable & PMRT with virtual bolus group & PMRT without virtual bolus group & $p$-Value \\
\hline $\mathrm{D}_{\max }(\mathrm{cGy})$ & $5571(5210-6222)$ & $5181(4861-5618)$ & $<0.01$ \\
$\mathrm{~S}_{5}\left(\mathrm{~cm}^{2}\right)$ & $619(473-942)$ & $583(436-891)$ & $<0.01$ \\
$\mathrm{~S}_{10}\left(\mathrm{~cm}^{2}\right)$ & $576(426-871)$ & $539(393-818)$ & $<0.01$ \\
$\mathrm{~S}_{20}\left(\mathrm{~cm}^{2}\right)$ & $508(370-772)$ & $464(331-704)$ & $<0.01$ \\
$\mathrm{~S}_{30}\left(\mathrm{~cm}^{2}\right)$ & $466(334-713)$ & $388(280-565)$ & $<0.01$ \\
$\mathrm{~S}_{40}\left(\mathrm{~cm}^{2}\right)$ & $421(296-662)$ & $142(51-229)$ & $<0.01$ \\
\hline
\end{tabular}

$\mathrm{D}_{\max }$ : Percentage of maximum dose; PMRT: post mastectomy radiotherapy; $\mathrm{S}_{5}$ : skin volume that received at least 5 Gy; $\mathrm{S}_{10}$ : skin volume that received at least $10 \mathrm{~Gy} ; \mathrm{S}_{20}$ : skin volume that received at least $20 \mathrm{~Gy} ; \mathrm{S}_{30}$ : skin volume that received at least $30 \mathrm{~Gy} ; \mathrm{S}_{40}$ skin volume that received at least 40 Gy.

grade 2 acute radiation dermatitis was 5311 cGy (sensitivity, $80 \%$; specificity, $83 \%$ ). The $\mathrm{D}_{\max }$ values in excess of the cutoff value of 5311 cGy were significantly higher in patients in the simulated plan group than in those in the real plan group $(p<0.01)$.

There were no significant differences in $\mathrm{D}_{2 \mathrm{cc}}, \mathrm{S}_{5}, \mathrm{~S}_{10}, \mathrm{~S}_{20}$, $S_{30}$, and $S_{40}$ between patients who developed grade 0-1 acute radiation dermatitis and those who developed grade 2 acute radiation dermatitis.

\section{Discussion}

This study showed that PMRT without bolus reduced the skin dose and resulted in no severe acute radiation dermatitis, and this was achieved without any increase in the local recurrence rates. In addition, according to the results of our DSH analysis, $\mathrm{D}_{\max }$ may be the most important factor for the development of acute radiation dermatitis.

In this study, only $4 \%$ of patients had local recurrences and $10 \%$ developed grade 2 acute radiation dermatitis, and these results were similar to those of Nakamura et al. (6) who found that after PMRT without bolus, $11 \%$ of patients had locoregional recurrence and $9 \%$ developed grade 2 acute radiation dermatitis.

Boluses are used to increase the dose for the chest-wall irradiation and to decrease the risk of local recurrence; however, radiation dermatitis is one of the most problematic toxicities associated with their use. Wright et al. (7) have reported that $80 \%$ of patients develop grade 2-3 radiation dermatitis after PMRT and the use of a bolus is a predictor for this complication. A direct comparison between PMRT with and without bolus by Uematsu et al. (8) revealed that the local recurrence rate was $5 \%$ in the PMRT with bolus group and $4 \%$ in the PMRT without bolus group, without significant difference, and our results are similar to these results in patients without severe acute radiation dermatitis.

Our DSH analysis revealed that $\mathrm{D}_{\max }$ contributes to the development of acute radiation dermatitis, and 5311 cGy was the cutoff value for the development of grade 2 acute radiation dermatitis. Chen et al. (9) have reported that a dose of 5,390 cGy within planning target volume was associated with a higher incidence of acute radiation dermatitis. This dose was similar to the cutoff value for the development of grade 2 acute radiation dermatitis in our study. In our study, PMRT without bolus lowered the $\mathrm{D}_{\max }$ than PMRT with bolus, which led to the lower frequency of grade 2 radiation dermatitis observed in this group. Therefore, $\mathrm{D}_{\max }$ might be contributing development of acute radiation dermatitis.

There were no significant differences in DSH parameters in $\mathrm{D}_{2 \mathrm{cc}}, \mathrm{S}_{5}, \mathrm{~S}_{10}, \mathrm{~S}_{20}, \mathrm{~S}_{30}$, and $\mathrm{S}_{40}$ between the patients who developed grade $0-1$ acute radiation dermatitis and those who developed grade 2 acute radiation dermatitis in this study, which may be because of the low frequency of grade 2 acute dermatitis observed. Lienger et al. (10) reported an association of radiation doses with acute radiation dermatitis, in that the doses from 40 to 65 Gy irradiated to $230-420 \mathrm{~cm}^{2}$ were associated with worse skin toxicities. In our data, the median $\mathrm{S}_{40}$ was $140 \mathrm{~cm}^{2}$ and no patients showed $\mathrm{S}_{40}$ that exceeded $230 \mathrm{~cm}^{2}$, which explains the lack of severe radiation dermatitis for $S_{40}$ and suggests that lowering $S_{40}$ is important to avoid severe acute radiation dermatitis.

In our study, locoregional recurrence was observed in two patients. One of these patients was a 41-year-old female patient who had a supraclavicular lymph node recurrence with invasive ductal carcinoma, the subtype was luminal B, and the Ki-67 positive rate was $70 \%$. Her radiation therapy plan was retrospectively reviewed and was found that the recurrence site on RTPS was irradiated with the prescribed dose of $\geq 95 \%$. The other patient, a 34-year-old female patient with invasive ductal carcinoma, had locoregional recurrence at the surgical wound site, the subtype was triple negative, and the Ki-67 positive rate was $80 \%-90 \%$. She was considered a patient with a high risk of recurrence because of her age, subtype, and $\mathrm{Ki}$ 67 positive rate $(11,12)$. Although we prescribe boost irradiation with electron beam only for patients with positive or close surgical margins, the boost irradiation may prevent recurrence in these high-risk patients. 
There were some limitations to our study. First, this was a retrospective study. Second, the use of various chemotherapy agents according to each patient may have affected the clinical outcomes of locoregional recurrence. However, no patient received concurrent chemotherapy during irradiation, and, therefore, the effects of chemotherapy on the acute radiation dermatitis can be considered small, if any.

In conclusion, chest-wall PMRT without bolus had the clinical advantage of reducing the skin dose and resulting in no severe acute radiation dermatitis without increasing the recurrence rates. In addition, the results of our DSH analysis showed that $\mathrm{D}_{\max }$ might be the most important factor contributing to the development of acute radiation dermatitis.

\section{Acknowledgements}

The Authors wish to thank all the patients who were involved in this study; the staff at the Division of Breast and Endocrine Surgery, Department of General Surgical Science, Gunma University Graduate School of Medicine; and our colleagues at the Department of Radiation Oncology, Gunma University Graduate School of Medicine.

\section{References}

1 McGale P, Taylor C, Correa C, Cutter D, Duane F, Ewertz M, Gray R, Mannu G, Peto R, Whelan T, Wang Y, Wang Z and Darby S: Effect of radiotherapy after mastectomy and axillary surgery on 10year recurrence and 20-year breast cancer mortality: meta-analysis of individual patient data for 8135 women in 22 randomised trials. Lancet (London, England) 383: 2127-2135, 2014.

$2 \mathrm{Vu}$ TT, Pignol JP, Rakovitch E, Spayne J and Paszat L: Variability in radiation oncologists' opinion on the indication of a bolus in post-mastectomy radiotherapy: an international survey. Clinical oncology (Royal College of Radiologists (Great Britain)) 19: 115-119, 2007

3 Pignol JP, Vu TT, Mitera G, Bosnic S, Verkooijen HM and Truong P: Prospective evaluation of severe skin toxicity and pain during postmastectomy radiation therapy. Int J Radiat Oncol Biol Phys 91: 157-164, 2015.

4 Yanagi T, Kamada T, Tsuji H, Imai R, Serizawa I and Tsujii H: Dose-volume histogram and dose-surface histogram analysis for skin reactions to carbon ion radiotherapy for bone and soft tissue sarcoma. Radiother Oncol 95: 60-65, 2010.
5 U.S. Department of Health and Human Services: Common terminology criteria for adverse events (CTCAE) version 4.0. National Institutes of Health, National Cancer Institute, 2009. Available from: https://www.eortc.be/services/doc/ctc/CTCAE_ 4.03_2010-06-14_QuickReference_5x7.pdf [Last accessed May $10,2018]$

6 Nakamura N, Arahira S, Zenda S, Yoneyama K, Mukai H, Onozawa M, Toshima M, Motegi A, Hirano Y, Hojo H, Kibe Y and Akimoto T: Post-mastectomy radiation therapy without usage of a bolus may be a reasonable option. J Radiat Res 58: 66-70, 2017.

7 Wright JL, Takita C, Reis IM, Zhao W, Lee E and Hu JJ: Racial variations in radiation-induced skin toxicity severity: data from a prospective cohort receiving postmastectomy radiation. Int $\mathbf{J}$ Radiat Oncol Biol Phys 90: 335-343, 2014.

8 Uematsu M, Bornstein BA, Recht A, Abner A, Come SE, Shulman LN, Silver B and Harris JR: Long-term results of postoperative radiation therapy following mastectomy with or without chemotherapy in stage I-III breast cancer. Int J Radiat Oncol Biol Phys 25: 765-770, 1993.

9 Chen MF, Chen WC, Lai CH, Hung $\mathrm{CH}$, Liu $\mathrm{KC}$ and Cheng YH: Predictive factors of radiation-induced skin toxicity in breast cancer patients. BMC cancer 10: 508, 2010.

10 Liegner L and Michaud N: Skin and subcutaneous reactions induced by supervoltage irradiation. Am J Roentgenol Radium Therapy Nuclear Med 85: 533-549, 1961.

11 Lim YJ, Lee SW, Choi N, Kwon J, Eom KY, Kang E, Kim EK, Kim JH, Kim YJ, Kim SH, Park SY and Kim IA: A novel prognostic nomogram for predicting risks of distant failure in patients with invasive breast cancer following postoperative adjuvant radiotherapy. Cancer Res Treat, 2017. doi: 10.4143/crt.2017.508. [Epub ahead of print]

12 Thangarajah F, Enninga I, Malter W, Hamacher S, Markiefka B, Richters L, Kramer S, Mallmann P and Kirn V: A retrospective analysis of Ki-67 index and its prognostic significance in over 800 primary breast cancer cases. Anticancer Res 37: 1957-1964, 2017.
Received April 25, 2018

Revised May 9, 2018

Accepted May 10, 2018 